\title{
A Case of Muscle Contraction-Induced Ischemic Limb Hyperemia in a Patient with Peripheral Arterial Disease during Incremental Repeat Isometric Knee Extensor Workloads
}

\author{
Takuya Osada ${ }^{1,2}$ \\ ${ }^{1}$ Cardiac Rehabilitation Center, Tokyo Medical University Hospital, Tokyo, Japan \\ ${ }^{2}$ Rehabilitation Center, Tokyo Medical University Hospital, Tokyo, Japan \\ Email: dentacmac@aol.com
}

How to cite this paper: Osada, T. (2020) A Case of Muscle Contraction-Induced Ischemic Limb Hyperemia in a Patient with Peripheral Arterial Disease during Incremental Repeat Isometric Knee Extensor Workloads. World Journal of Cardiovascular Diseases, 10, 796-808.

https://doi.org/10.4236/wjcd.2020.1012076

Received: November 27, 2020

Accepted: December 26, 2020

Published: December 29, 2020

Copyright $\odot 2020$ by author(s) and Scientific Research Publishing Inc. This work is licensed under the Creative Commons Attribution International License (CC BY 4.0).

http://creativecommons.org/licenses/by/4.0/

\begin{abstract}
Background: To determine whether muscle contraction-induced leg blood flow (LBF) during exercise may be altered in a patient with an ischemic limb due to peripheral arterial disease (PAD) compared with the non-PAD limb. Case Presentation: A 66-year-old male patient with intermittent claudication due to PAD in the right leg (ankle brachial pressure index, 0.69) showed complete obstruction in both common iliac arteries including internal/external segments with collaterals above the femoral artery and popliteal artery with collaterals, and in the healthy left non-PAD-leg (1.06). He attempted unilateral repeat isometric knee extensions at a target contraction rhythm with each leg at incremental contraction intensities $(5 \%, 10 \%$, and $30 \%$ of maximum voluntary contraction [MVC] for $3 \mathrm{~min}$ at each intensity). Blood velocity/flow (Doppler ultrasound) in the femoral artery, blood pressure, and leg vascular conductance (LVC) were measured. Isometric thigh MVC strength pre-exercise was similar between the PAD-leg $(48.0 \mathrm{~kg})$ and non-PAD-leg (48.7 kg). Pre-exercise LBF $(\mathrm{ml} / \mathrm{min})$ was also similar between the PAD-leg (316) and non-PAD-leg (327). Blood pressure increases were similar during exercise. Average exercising LBF in $\mathrm{ml} / \mathrm{min}$ in the last $1 \mathrm{~min}$ at each intensity was higher in the PAD-leg than the non-PAD-leg: 1087 vs. 471 at 5\%, 2097 vs. 712 at $10 \%$, and 2656 vs. 1517 at $30 \%$ MVC with a close positive linear relationship between LBF and \%MVC in the non-PAD-leg $(r=0.999, \mathrm{P}<0.01)$, in agreement with previous findings, but less significant in the PAD-leg $(\mathrm{r}=$ $0.879, \mathrm{P}=\mathrm{NS}$ ), indicating intense vasodilation (increasing LVC) in the PAD-leg compared with the non-PAD-leg. Conclusion: Knee extensor exercising LBF in the femoral artery was dissimilar between the PAD-leg and non-PAD-leg at the same exercise intensity, even though pre-exercising LBF
\end{abstract}


was the same. Further research on the time-course in hemodynamics during leg exercise in PAD might potentially provide insight for the cardiovascular adjustment in severity of arteriosclerosis, stenosis and/or collaterals reserve.

\section{Keywords}

Leg Blood Flow, Peripheral Arterial Disease, Collaterals Flow, Repeat Isometric Knee Extensor Exercise, Doppler Ultrasound

\section{Introduction}

Peripheral arterial disease (PAD), resulting in intermittent claudication, is associated with low exercise tolerance [1]. This can reduce levels of daily physical activity (for example, low walking ability), lead to poor health, and impair quality of life [2]. Long-term, habitual exercise improves walking distance with less pain in the leg [3] [4]. This may potentially be due to alteration (or adjustment) of the peripheral hemodynamics in the leg and/or improvement in the muscle oxidative capacity or potential oxygen supply via developed collateral circulation by exercise training [5]. Therefore, it might be important to understand the role of the blood flow circulatory effect related to voluntary muscle contractions such as knee and/or plantar exercise thorough hemodynamic imaging using real-time recording with a non-invasive method.

In 1976, Nicolaides reported the ability of blood velocity tracing in the femoral artery to predict the state of the proximal aortoiliac segment in PAD. Furthermore, an illustration of changes in femoral artery blood velocity after exercise was described [6]. There have been numerous previous studies on the time course of muscle contraction-induced blood flow hyperemia in the limb conduit artery due to exercise in healthy subjects [7], but less acknowledgement of the reference values for exercising blood flow in the leg conduit artery with lesion of vascular disease.

Recent advances in ultrasonography with two-dimensional anatomical imaging of monitored pulse flow and Doppler waveform can precisely evaluate arterial lesions. Thus, the measurement of (changes in) blood velocity profile can provide valuable information on the severity of stenosis due to arteriosclerosis as well as existence of collaterals induced by ischemia with obstruction of artery flow and to evaluate alterations in the magnitude of peak systolic blood velocity and/or diastolic blood velocity profile in a basal non-exercising state in PAD [8].

In addition, Doppler imaging can provide high temporal resolution of blood velocity in a conduit artery at rest. Moreover, rapid changes in blood velocity in a conduit artery located in a gap into a major muscle group can also be detected with muscle contraction and relaxation and/or cardiac beat-by-beat during exercise, muscle contraction frequency and workload, and in relation to vasodilatation/vasoconstriction [9] [10] [11] [12]. In previous studies in healthy legs on exercise leg blood flow (LBF), there were positive linear correlations between 
unilateral LBF and workload during steady state dynamic leg exercise with a validated operator technique [13] [14] [15] [16].

Limited information is available on whether the muscle contraction-induced ischemic limb flow response in PAD may be altered during leg exercise. Thus, in the present case with PAD, we sought to determine the magnitude of thigh muscle strength-dependent blood flow in the femoral artery located above an arteriosclerotic lesion using Doppler ultrasound.

\section{Case Presentation}

A male (66 yr 3 mo, $173.8 \mathrm{~cm}, 69.5 \mathrm{~kg}$ ) had been diagnosed 5 years previously with hypertension and PAD in the right leg with intermittent claudication (Fontaine classification II). The intermittent claudication had appeared 2 years prior to the study. In the patient's right leg there was a region of complete obstruction due to arteriosclerosis in the right common iliac artery, including the internal-external iliac artery and the developed collaterals above the common femoral artery as well as complete obstruction of the popliteal artery with collaterals, as determined by angiography, and the patient's left leg was healthy and without PAD. Ankle brachial pressure index (ABI) was 0.69 for the PAD-leg and 1.06 for the non-PAD-leg (CAVIpluse VaSera VS-1000, Fukuda Denshi, Tokyo, Japan). A normal ABI is defined as a resting measurement greater than 0.9. Any value of 0.9 or less indicates the presence of $\mathrm{PAD}$, with lower $\mathrm{ABI}$ values indicating more severe PAD. Patients with ABI values of 0.70 to 0.90 may be asymptomatic or have very mild symptoms of intermittent claudication [17].

The patient had been taking oral cilostazol, an antiplatelet agent with vasodilatory properties, and valsartan, as an antihypertensive agent. He has continued hospital-based rehabilitation with 30-min aerobic bicycle ergometer exercise approximately 2 times per week for 2 years 3 months. Cardiopulmonary ergometer exercise tests indicated a maximum oxygen consumption of $21.69 \mathrm{ml} / \mathrm{kg} / \mathrm{min}$ (6.2 Mets), with a maximum workload as 121 watts and maximum heart rate of 141 beat per minute. The present trial was conducted according to the principles of the Declaration of Helsinki (1964) and with approval (approval No. 958) of the Institutional Ethics Committee of our institution. The patient gave written consent and was informed of the nature and purpose of the study, as well as potential risks and discomfort. The patient also understood that they could withdraw from the study at any time without consequence.

\section{Protocol}

\subsection{Exercise Model}

The patient performed incremental unilateral (one-legged) isometric knee extensor exercise in a sitting position, which is an appropriate model for the determination of comprehensive LBF in the femoral artery during limb muscle contractions (Figure 1). This exercise model allows stable measurements of femoral arterial blood velocity using Doppler ultrasound, whereas usual walking/running models 


\section{5-s isometric knee extension Contraction phase}

\section{5-s relaxation pause Non-contraction phase}

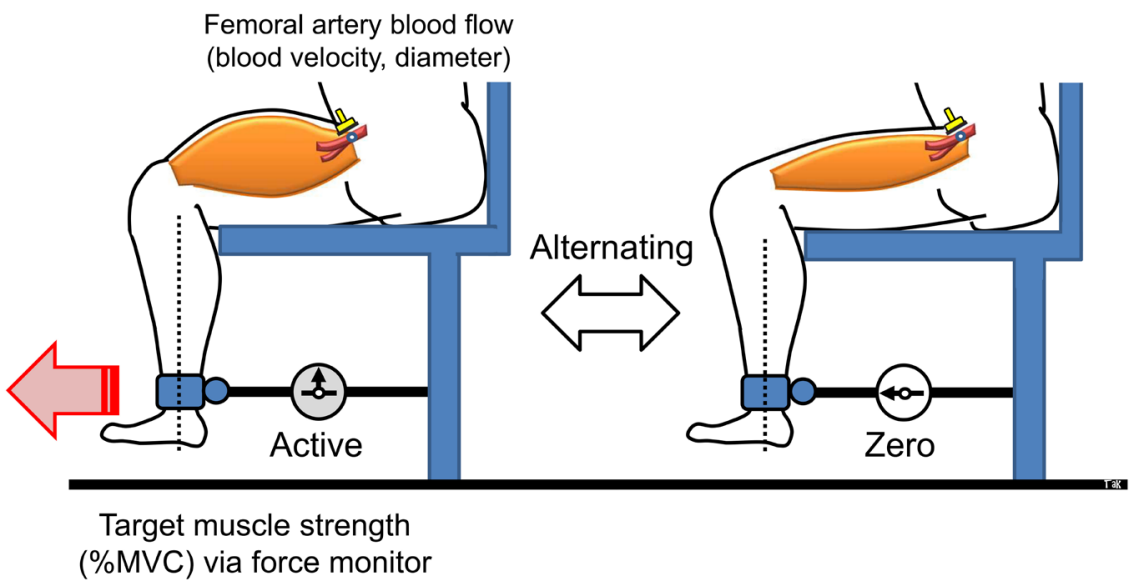

Figure 1. One-legged knee extensor exercise model. Repeat isometric muscle contraction performed as 5-s voluntary (active) isometric knee muscle contraction and 5-s muscle relaxation pause (10 s/duty cycle) for $3 \mathrm{~min}$ at 5\%, 10\%, 30\%, and 50\% MVC. The knee extensor contraction rhythm was maintained by following the pace of a visible and audible metronome. The knee extensor contraction strength (target intensity) was performed by visualizing the contraction strength displayed in real time on a force monitor. Simultaneous recording of hemodynamic parameters was measured for the whole experiment.

do not easily allow LBF measurement in the leg conduit artery, due to the difficulty of fixing the scanning probe to the hip joint [16] [18] [19] [20]. The patient performed exercise with the hips at a $100^{\circ}$ angle, the thigh positioned horizontally with the knee joint bent at an approximately $110^{\circ}$ angle, and their foot and ankle secured to upper and lower rods with the use of a custom-designed Meiko-100 knee-extension ergometer (Meiko Co. Ltd., Tokyo, Japan) [13].

\subsection{Exercise Intensity and Muscle Contraction-Relaxation Frequency}

Prior to exercise, the maximum voluntary contraction force (MVC) expressed as the maximum muscle strength throughout a single muscle contraction bout in each leg was measured using a knee-extension ergometer connected to a strain-gauge (Meiko Co. Ltd. ST-200, Tokyo, Japan). The MVC was determined by the averaging over five repetitions for a bolus of MVC. The target intensity at isometric muscle contraction phase increased every 3 min corresponding to $5 \%, 10 \%$, $30 \%$, and $50 \%$ MVC. The recovery phase lasted $10 \mathrm{~min}$ (Figure 2). A duty cycle for muscle contraction-relaxation was at a rate of 5-s isometric knee extensor contractions at the target exercise intensity and consequently 5 -s muscle relaxation (pause) corresponding to a cycle of $10 \mathrm{~s}$ (6 cycles per minutes) with recording of the muscle strength curve. The patient attempted to maintain the target intensity using a digital visualization of the intensity displayed in real time on a monitor. The duration of muscle contraction and muscle rhythm followed the pace of an audible metronome (Quartz Metronome SQ 70, SEIKO, Tokyo Japan). The patient performed the exercise until they reached all-out exhaustion with leg fatigue. 


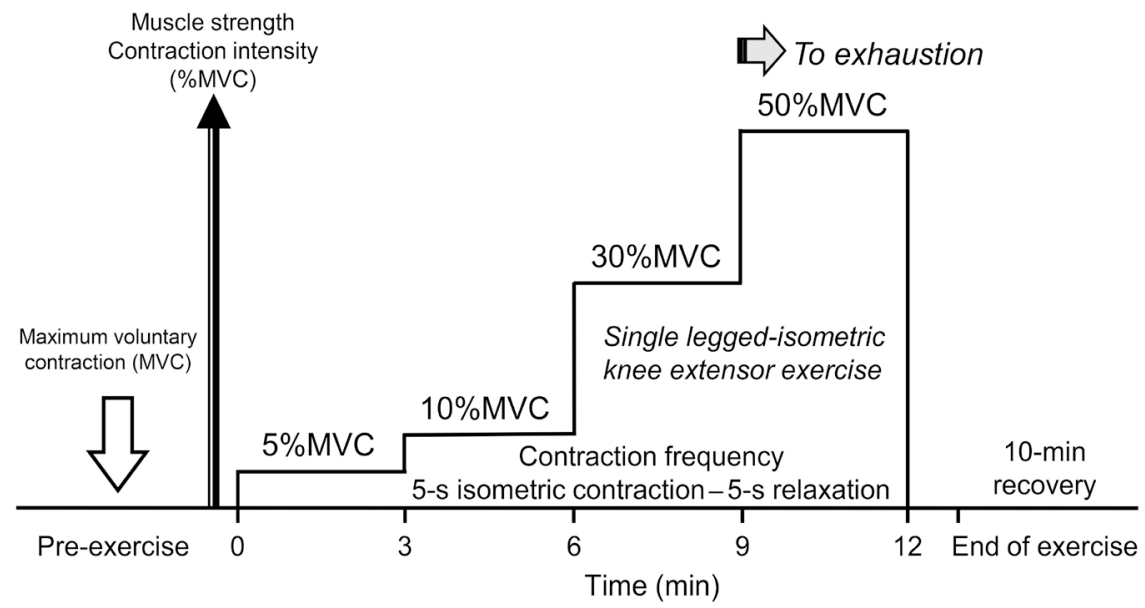

Figure 2. Exercise protocol. The maximum voluntary contraction (MVC) strength was measured for each leg at pre-exercise. There were four different target exercise intensities in the multi-stage incremental unilateral isometric knee extensor exercise sessions, 5\%, $10 \%, 30 \%$, and $50 \%$ of MVC, and each target intensity for $3 \mathrm{~min}$. A 10 -min recovery was taken after the end of exercise. The participant attempted to exercise to exhaustion with leg fatigue.

\subsection{Blood Velocity and Vessel Diameter in the Femoral Artery}

An ultrasonograph (SONOS 1500, ultrasound imaging system, HP 77035A, Hewlett Packard, Tokyo Japan) with a 2-dimensional ultrasonic imager and a pulse Doppler flowmeter using linear array prove $(7.5 \mathrm{MHz})$ was used. Measurement in the proximal femoral artery was at a site with minimum turbulence and without influence of the inguinal region on hemodynamics above the bifurcation, thereby enabling easy and reliable measurement during leg exercise [10] [11] [12] [13]. In the present patient, there was no pathology (severe stenosis or collateral vessel) in the target femoral artery (sampling point) monitoring arterial pulsation color images above the bifurcation into the superficial and profunda femoral branch (see 2-D image for PAD in Figure 3). Validated blood velocity measurement in the femoral artery has been reported previously during repeated muscle contractions using Doppler ultrasound [9] [10] [11].

Prior to exercise, the vessel diameter at pre-exercise (basal state) was measured only for determination of the cross-sectional area. The systolic and diastolic vessel diameters were also measured under perpendicular insonation and calculated in relation to the temporal duration of the ECC recording curve as [(systolic vessel diameter $\times 1 / 3)+($ diastolic vessel diameter $\times 2 / 3)$ ] [9] [10] [11]. LBF was calculated by multiplying the cross-sectional area [area $=\pi \times$ (vessel diameter $/ 2)^{2}$ ] by mean blood velocity.

\subsection{Blood Pressure, Heart Rate and Leg Vascular Conductance}

Blood pressure was monitored continuously using an auricular plethysmography device with oscillometric calibration, with a cuff tourniquet placed on the upper arm (RadiaPress RBP-100, KANDS, Aichi, Japan) with data stored using a 


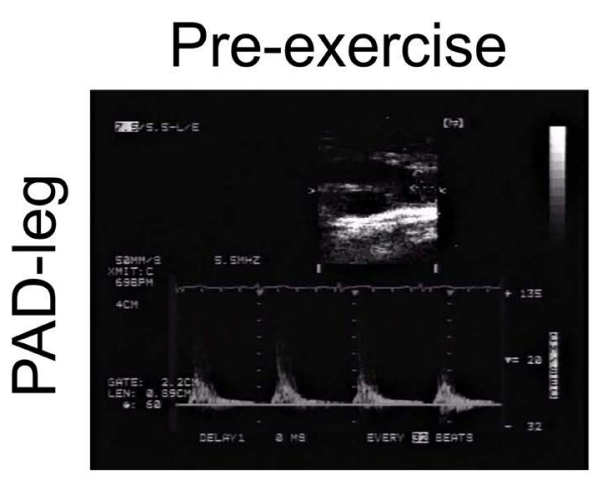

\section{$30 \% \mathrm{MVC}$ exercise}
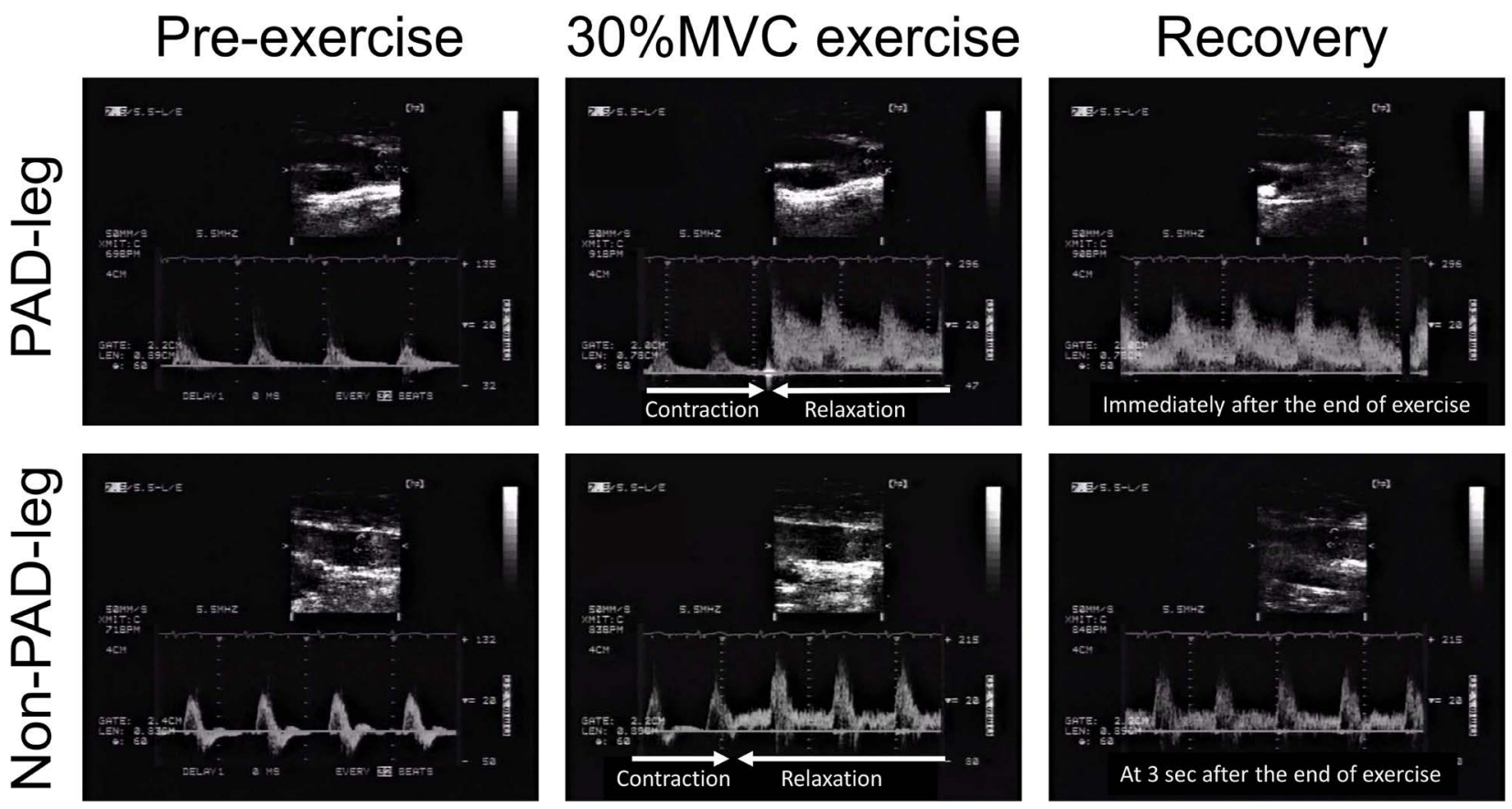

Figure 3. Comparison in blood velocity profile in femoral artery between PAD and non-PAD. The blood velocity in the femoral artery above the bifurcation monitored in B-mode was clearly different between the PAD-leg (a monophasic pattern with low resistance component) and the non-PAD-leg (normal triphasic pattern) at pre-exercise in the left panel. The upper panels are for the PAD-leg and the lower panels for the non-PAD-leg. The blood velocity profile showed the restricted blood flow due to increases in intramuscular pressure during muscle contraction $(\rightarrow)$, and hyperemic increasing blood flow during muscle relaxation $(\leftarrow)$. Marked higher blood velocity in the diastolic component was obviously seen in the PAD-leg than the non-PAD-leg at $30 \% \mathrm{MVC}$ in the middle panel (note the higher scale on the vertical axis in the PAD-leg compared with the non-PAD-leg). The magnitude of the hyperemic state after the end of exercise was enhanced in the PAD-leg compared with the non-PAD-leg in the right panel. PAD: peripheral arterial disease, \%MVC: percentage of maximum voluntary contraction.

PowerLab data acquisition system (Chart v.4.2.3 software; AD Instruments, Sydney, Australia). Heart rate was measured using the beat-by-beat from continuous recording of the blood pressure wave. Leg vascular conductance was calculated as LBF divided by blood pressure ( $\mathrm{LBF} / \mathrm{blood}$ pressure) using the unit $\mathrm{ml} / \mathrm{min} / \mathrm{mmHg}$.

\subsection{Sampling Collections and Evaluations}

We collected the samples for hemodynamic measures at pre-exercise and during exercise (every 5-s muscle contraction phase, every 5-s muscle relaxation phase, and every 10-s muscle contraction-relaxation duty cycle) and recovery (immediately at the end of the exercise point, and every $1 \mathrm{~min}$ ). Furthermore, the average LBF value during steady state exercise was also determined as a mean value (6th duty muscle contraction-relaxation cycles) in the last $1 \mathrm{~min}$ of exercise at each exercise intensity. Statistical comparisons were examined using a linear fitting regression correlation coefficient ( $r$ ), and $\mathrm{P}$-value were conducted between mean LBF and the mean muscle contraction strength (relative value as \%MVC) in the last $1 \mathrm{~min}$ of exercise at each exercise intensity (Microsoft 365 Excel). A $\mathrm{P}$-value $<0.05$ was considered significant. 


\section{Results}

ABI was lower in the ischemic PAD-leg (0.69) than the healthy non-PAD-leg (1.06), which may indicate the existence of stenosis and/or obstruction in the limb artery below 0.9 . The MVC in one-legged isometric knee extensor muscle contraction was similar between the PAD-leg $(48.0 \mathrm{~kg})$ and non-PAD-leg $(48.7 \mathrm{~kg})$, which indicated the same relative target muscle contraction intensity (\%MVC). The pre-exercise $\mathrm{LBF}$ in a basal resting state was similar between $316 \mathrm{ml} / \mathrm{min}$ in the PAD-leg and $327 \mathrm{ml} / \mathrm{min}$ in the non-PAD-leg. In the PAD-leg in a pre-exercise basal state there was a monophasic blood velocity profile, but a triphasic blood velocity pattern was seen in the non-PAD-leg (Figure 3). The duration of unilateral leg exercise for achieving to exhaustion (leg fatigue but not intermittent claudication) was shorter in the PAD-leg at $10 \mathrm{~min}$ than the non-PAD-leg at $11 \mathrm{~min} 40 \mathrm{sec}$ and it was not to possible to accomplish the whole session at 50\% MVC.

The magnitude of the average LBF was higher in the PAD-leg than the non-PAD-leg during exercise (Figure 4). The average exercise LBF in $\mathrm{ml} / \mathrm{min}$ in the last $1 \mathrm{~min}$ of each exercise intensity was higher in the PAD-leg than the non-PAD-leg: 1087 vs 471 at 5\% MVC, 2097 vs 712 at 10\% MVC, and 2656 vs 1517 at 30\% MVC in Figure 4A, which may indicate excess vasodilation (increasing LVC) in the PAD-leg compared with the non-PAD-leg in Figure 4B. The increase in blood pressure was similar in the PAD-leg and non-PAD-leg during exercise (Figure 4C). The increase in heart rate was similar between the PAD-leg and non-PAD-leg in Figure 4D, which can be used to validate the physiological response using the same muscle contraction strength (Figure 4E).

The LBF in both legs increased in a manner almost dependent on muscle contraction intensity, furthermore the non-PAD-leg showed that the increase in LBF was statistically significant $(\mathrm{r}=0.999, \mathrm{P}<0.01)$ with positive linearity by \%MVC, but not in the PAD-leg $(r=0.879, \mathrm{P}=\mathrm{NS})$ (Figure 5).

\section{Discussion}

The present trial may provide unique insights on patients with PAD that pathophysiologically have a limitation in perfusion blood flow to skeletal muscles in the periphery, although this result is only for one patient without consideration of the reserve capacity in the vasculature (oxygen delivery compensated by collateral flow, etc.) following long-term morbidity with impairment of the blood flow pathway between upstream and downstream and/or other interventions such as medication effectiveness or exercise therapy (changes in exercise tolerance and/or muscle strength).

\subsection{Muscle Contraction-Induced Hyperemia in PAD and Non-PAD}

Impairment from stenosis and atherosclerosis due to an obstructed conduit artery in PAD should functionally be a limitation in perfusion flow via the muscle capillary, and there have been recent report evaluating the microcirculation using contrast-enhanced ultrasound [21] [22] [23] and oxygenation using near-infrared 

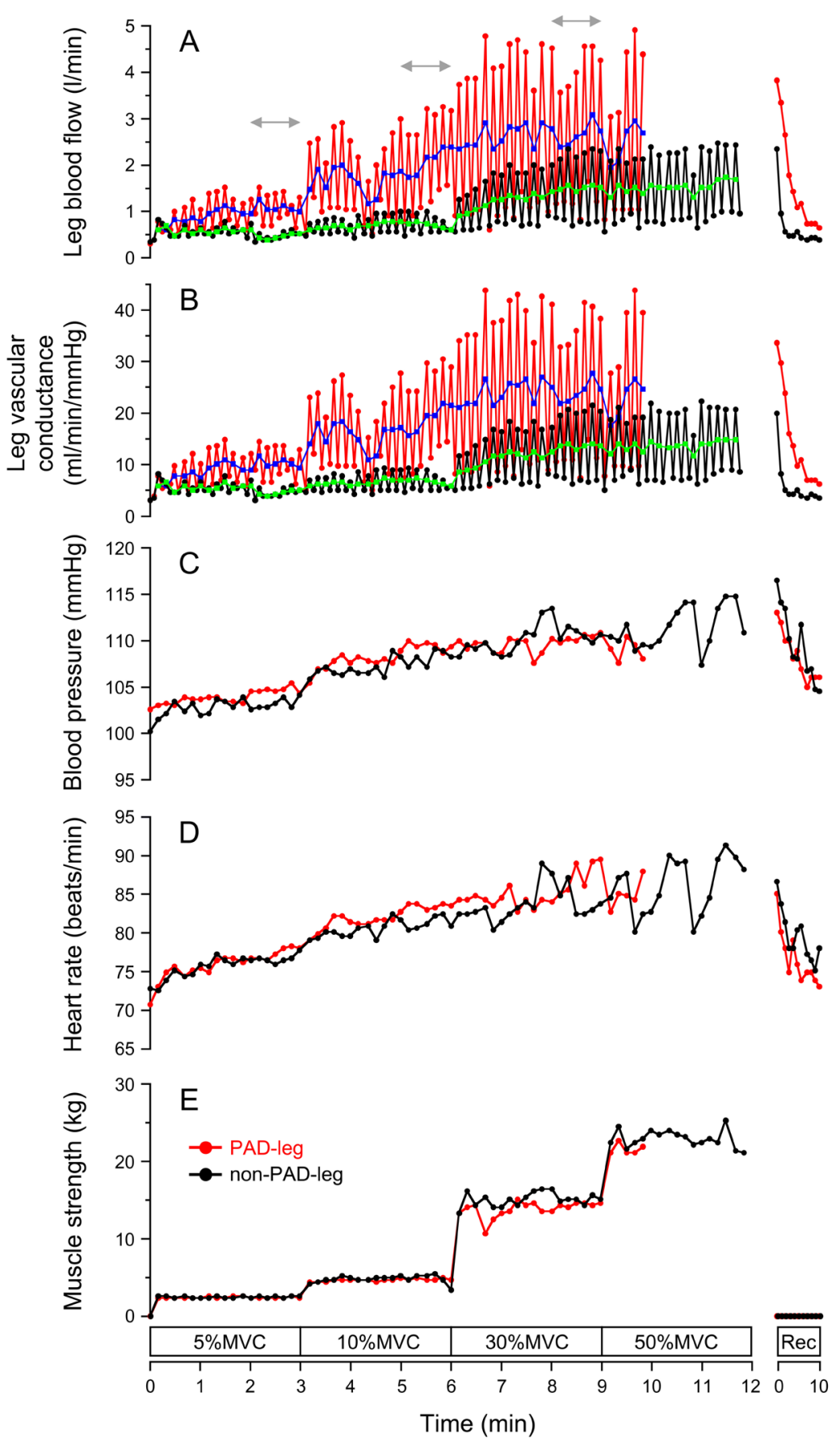

Figure 4. Time-course of hemodynamics parameter at rest, during exercise, and recovery. The time-course in LBF (A) as well as LVC (B) was higher in the PAD-leg (red line) than the non-PAD-leg (black line). The oscillation in LBF indicated restricted flow during muscle contraction (below circles) and non-restricted flow during muscle relaxation (above circles). Average value of muscle contraction and relaxation in a duty cycle appeared rectangular in the PAD-leg (blue color) and the non-PAD-leg (green color). Average value of blood pressure (C) and heart rate (D) determined by muscle contraction and relaxation in a duty cycle during exercise were similar between the PAD-leg and non-PAD-leg. In both the PAD-leg and the non-PAD-leg, the muscle strength corresponding to target intensity as relative muscle strength (\%MVC) was stable in each voluntary isometric muscle contraction (E). Muscle strength during muscle relaxation was not plotted because the value was zero. Muscle strength was almost same at each contraction intensity (\%MVC) because of similarity in MVC between the PAD-leg (48.0 kg) and non-PAD-leg (48.7 kg). Average LBF during last $1 \mathrm{~min}$ of steady state exercise $(\leftrightarrow)$ was used for the relationship with \%MVC in Figure 5. LBF: leg blood flow, LVC: leg vascular conductance, PAD: peripheral arterial disease, \%MVC: percentage of maximum voluntary contraction. 


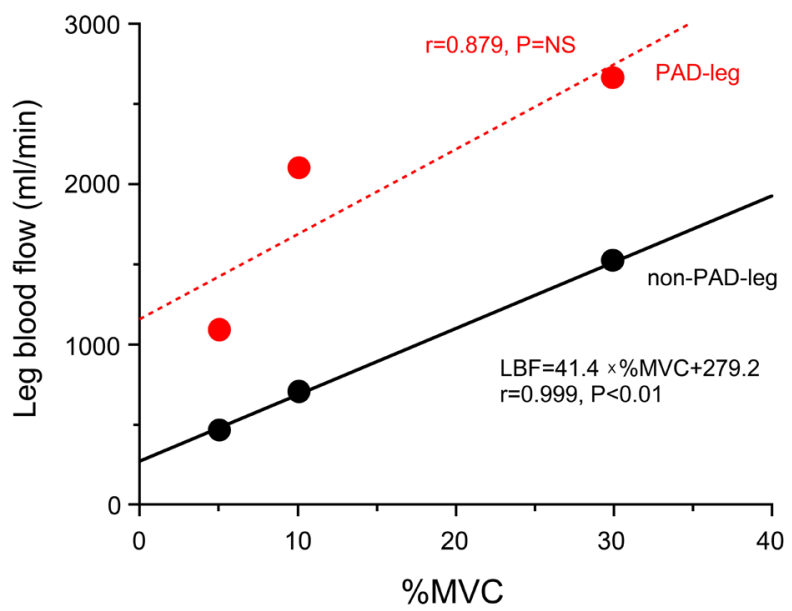

Figure 5. Relationship between LBF and \%MVC during exercise. There was a statistically significant $(\mathrm{r}=0.999, \mathrm{P}<0.01$ in black solid line) positive linear relationship between LBF and \%MVC in the non-PAD-leg despite fewer sample, but no significance in the PAD-leg ( $r=0.879, \mathrm{P}=\mathrm{NS}$ in dashed red line). The LBF at 50\% MVC was not included because the whole exercise secession was not completed. LBF: leg blood flow, \%MVC: percentage of maximum voluntary contraction, PAD: peripheral arterial disease.

spectroscopy [24] in the lower limb muscles during exercise. These studies investigated the relationship between muscle metabolism and perfusion flow in the vascular bed because PAD is associated with severe exercise intolerance related to impaired endothelial function and/or alterations in skeletal muscle phenotype rather than hemodynamic impairment in the conduit artery.

On the other hand, during exercise the magnitude of hemodynamics in the leg conduit artery from the upper stream around the lesion segment in PAD may speculatively detect any physiological changes due to remodeling of the peripheral circulation via development of significant collaterals with a change in chronic ischemic state over time.

In fact there have been few previous reports to evaluate the time-course of exercising LBF in a conduit artery coordinated using precise repeat voluntary muscle contraction strength (every muscle contraction-relaxation duty cycle) even if the flow profile in the sampling area might be directly influenced by the collaterals surrounding the obstructive lesion connected to the upper stream and/or downstream during exercise. Possible reasons for the paucity of reports are that it may not be easy to quantify and/or quality the role of comprehensive blood flow supply via the development of collaterals from the upper stream above the lesion to downstream with an intervention such as exercise therapy, medication, and/or the collateral development in a cohort study.

The spectrum of lesions in the leg arteries in PAD with claudication is varied. A long segmental obstruction can coexist with almost normal arterial segments [8]. Thus, it was acceptable that the present patient also had a lesion in the right common iliac artery including the internal-external iliac artery and the developed collaterals above the common femoral artery as well as complete obstruction of the popliteal artery with collaterals, whereas almost normal arteries in the 
left leg showed a normal $A B I$ range.

In a present case with the above-mentioned PAD affecting a unilateral leg, there was a clear difference in exercise LBF during incremental repeat isometric knee extension between the PAD-leg and non-PAD-leg despite LBF in the basal pre-exercise state being similar (Figure 4). The mechanism of muscle contraction-induced hyperemia in the PAD-leg compared with the non-PAD-leg shown in a present case was naturally unclear in the cross-sectional analysis.

The present characteristics in both leg hemodynamics might be acceptable because of the statistically significant $(\mathrm{P}<0.01, \mathrm{n}=3)$ relationship between LBF and \%MVC as non-PAD leg in Figure 5 in agreement with our previous finding in healthy participants [11] [12]. Moreover, accurate performance of each leg exercise at the precise target contraction intensities (almost same MVC both leg) may give credible data with a uniform oscillation in LBF value influenced by the fluctuation in each muscle contraction strength (Figure 4E). Furthermore, during exercise the physiological cardiovascular response both blood pressure and heart rate was almost similar between the PAD-leg and non-PAD-leg (Figure 4C and Figure 4D).

Exercise hyperemia with vasodilation is related to intrinsic (endothelial-related factors, autacoid substances, metabolite and myogenic response) as well as extrinsic (autonomic nerve regulation, signal/reflexes with central command and exercise pressor reflexes with mechanical muscle contraction/accumulated metabolite product) regulation, as well as changes in arteriovenous pressure gradient due to the mechanical muscle pump.

Thus, the promoted hyperemic state in the present PAD case can speculatively be expressed as vessel dilation in the vascular bed due to multiple above-mentioned factors enhanced by collaterals, medication control (vasodilatory properties in disease leg), and/or long term of habitual exercise training [17].

\subsection{Changes in Doppler Waveform during Exercise}

$\mathrm{PAD}$ is often diagnosed by noting a change in the blood velocity pattern (waveforms) on the Doppler spectrum sampled above or distal to the site of an arterial lesion. The artery blood velocity profile in a proximal region may appear normal; however, the downstream blood velocity profile will typically show a monophasic pattern, with a low resistance component if there is sufficient vasodilation. Moreover, the monophasic pattern showed low-systolic forward flow velocities persists during the cardiac cycle [8].

Furthermore, significant hemodynamics in the arterial lesion cause a period of early diastolic flow reversal (backward negative flow) to decrease and ultimately disappear as the lesion becomes more severe; consecutively the late diastolic component of the forward flow increases in magnitude as the severity of the proximal lesion worsens [8].

In Figure 3 at pre-exercise, the blood velocity profile in the present PAD patient was in agreement with such alterations in waveform, which is a loss of 
backward flow at the second dicrotic notch as well as slight increase in forward flow at the end-diastolic phase. In addition, the peak systolic blood velocity was lower in the PAD-leg (approximately $40 \mathrm{~cm} / \mathrm{s}$ ) than the non-PAD-leg (approximately $60 \mathrm{~cm} / \mathrm{s}$ ).

These changes may represent a combination of factors such as progressive dilatation and recruitment of peripheral arterioles within the distal vascular bed of the leg as well as the development of many small collateral branches, which correspond to the surrounding popliteal artery in the present patient.

During repeat isometric muscle contraction exercise, the peak systolic blood velocity increased in both the PAD-leg and the non-PAD-leg, although a restricted blood velocity profile was shown due to high intramuscular pressure without changes in blood velocity in the diastolic phase. Conversely, the blood velocity profile increased dramatically in both systolic and diastolic components during muscle relaxation with strong vasodilation in the entire diastolic component in both the PAD-leg and non-PAD-leg (see 30\% MVC in Figure 3). The higher blood velocity profile in the entire cardiac cycle was notably detected in the PAD-leg than the non-PAD-leg. The post exercise hyperemic state after the end of exercise, the blood velocity declined as exponential decay during recovery in Figure 4A.

Doppler ultrasound can non-invasively detect with beat-to-beat high resolution the temporal pulsatile blood velocity profiles in the conduit artery at rest as well as during muscle contractions for a patient with PAD.

\section{Conclusion}

This is an initial case trial in PAD for the determination of a time-course in LBF in the femoral artery with surrounding collaterals during voluntary thigh muscle contractions with knee extensor exercise using Doppler ultrasound. To investigate rapid changes in exercise LBF in the conduit artery with the obstructive lesion in the vasculature above and/or below may be a new insight for consideration of cardiovascular remodeling as a collateral flow with hyperemic state due to transient exercise and/or possible effectiveness due to hospital-based rehabilitation. Further research on the peripheral circulatory response due to exercise is necessary in PAD.

\section{Acknowledgements}

The author acknowledges the support of the late professor emeritus Atsuko Kagaya of Japan Women's College of Physical Education, the late professor emeritus Bengt Saltin of the CMRC and the late professor emeritus Hisao Iwane formerly of The Tokyo Medical College for contributions leading to the present state of clinical physiology for rehabilitation. This study was supported in part by the "Academic Frontier" Project for Private Universities (JWCPE), 2004-2008, and a Grant-in-Aid for Scientific Research (C) in Scientific Research (No. 19500617) from the MEXT in Japan and the JSPS, 2007-2008 (to T. Osada). 


\section{Conflicts of Interest}

The author declares no conflicts of interest regarding the publication of this paper.

\section{References}

[1] Askew, C.D., Green, S., Walker, P.J., Kerr, G.K., Green, A.A., Williams, A.D. and Febbraio, M.A. (2005) Skeletal Muscle Phenotype Is Associated with Exercise Tolerance in Patients with Peripheral Arterial Disease. Journal of Vascular Surgery, 41, 802-807. https://doi.org/10.1016/j.jvs.2005.01.037

[2] Hiatt, W.R. (2001) Medical Treatment of Peripheral Arterial Disease and Claudication. New England Journal of Medicine, 344, 1608-1621. https://doi.org/10.1056/NEJM200105243442108

[3] Hiatt, W.R., Regensteiner, J.G., Hargarten, M.E., Wolfel, E.E. and Brass, E.P. (1990) Benefit of Exercise Conditioning for Patients with Peripheral Vascular Disease. Circulation, 81, 602-609. https://doi.org/10.1161/01.CIR.81.2.602

[4] Larsen, O.A. and Lassen N.A. (1966) Effect of Daily Muscular Exercise in Patients with Intermittent Claudication. The Lancet, 288, 1093-1096.

https://doi.org/10.1016/S0140-6736(66)92191-X

[5] Stewart, K.J., Hiatt, W.R., Regensteiner, J.G. and Hirsch, A.T. (2002) Exercise Training for Claudication. New England Journal of Medicine, 347, 1941-1951. https://doi.org/10.1056/NEJMra021135

[6] Nicolaides, A.N., Gordon-Smith, L.C., Dayandas, J. and Eastcott, H.H.G. (1976) The Value of Doppler Blood Velocity Tracings in the Detection of Aortoiliac Disease in Patients with Intermittent Claudication. Surgery, 80, 774-778.

[7] Joyner, M.J. and Casey, D.P. (2015) Regulation of Increased Blood Flow (Hyperemia) to Muscles during Exercise: A Hierarchy of Competing Physiological Needs. Physiological Review, 95, 549-601. https://doi.org/10.1152/physrev.00035.2013

[8] Polak, J.F. (2004) Peripheral Vascular Sonography. A Practical Guide. Second Edition, Lippincott Williams \& Wilkins, Philadelpha.

[9] Rådegran, G. (1997) Ultrasound Doppler Estimates of Femoral Artery Blood Flow during Dynamic Knee Extensor Exercise in Humans. Journal of Applied Physiology, 83, 1383-1388. https://doi.org/10.1152/jappl.1997.83.4.1383

[10] Osada, T. (2004) Muscle Contraction-Induced Limb Blood Flow Variability during Dynamic Knee Extensor. Medicine \& Science in Sports \& Exercise, 36, 1149-1158. https://doi.org/10.1249/01.MSS.0000132272.36832.6A

[11] Osada, T. and Rådegran, G. (2002) Femoral Artery Inflow in Relation to External and Total Work Rate at Different Knee Extensor Contraction Rates. Journal of Applied Physiology, 92, 1325-1330. https://doi.org/10.1152/japplphysiol.00848.2001

[12] Osada, T. and Rådegran, G. (2009) Femoral Artery Blood Flow and Its Relationship to Spontaneous Fluctuations in Rhythmic Thigh Muscle Workload. Clinical Physiology and Functional Imaging, 29, 277-292. https://doi.org/10.1111/j.1475-097X.2009.00868.x

[13] Osada, T., Katsumura, T., Hamaoka, T., Inoue, S., Esaki, K., Sakamoto, A., Murase, N., Kajiyama, J., Shimomitsu, T. and Iwane, H. (1999) Reduced Blood Flow in Abdominal Viscera Measured by Doppler Ultrasound during One-Legged Knee Extension. Journal of Applied Physiology, 86, 709-719.

https://doi.org/10.1152/jappl.1999.86.2.709

[14] Shoemaker, J.K., Hodge, L. and Hughson, R.L. (1994) Cardiorespiratory Kinetics 
and Femoral Artery Blood Velocity during Dynamic Knee Extension Exercise. Journal of Applied Physiology, 77, 2625-2632. https://doi.org/10.1152/jappl.1994.77.6.2625

[15] Hughson, R.L., Shoemaker, J.K., Tschakovsky, M.E. and Kowalchuk, J.M. (1996) Dependence of Muscle VO2 on Blood Flow Dynamics at Onset of Forearm Exercise. Journal of Applied Physiology, 81, 1619-1626.

https://doi.org/10.1152/jappl.1996.81.4.1619

[16] Osada, T., Saltin, B., Mortensen, S.P. and Rådegran, G. (2012) Measurement of the Exercising Blood Flow during Rhythmical Muscle Contractions Assessed by Doppler Ultrasound: Methodological Considerations. Journal of Biomedical Science and Engineering, 5, 779-788. https://doi.org/10.4236/jbise.2012.512A098

[17] Hiatt, W.R., Hirsch, A.T. and Regensteiner, J.G. (2001) Peripheral Arterial Disease. CRC Press, Boca Raton.

[18] Osada, T., Saltin, B. and Rådegran, G. (2013) Assessment of Voluntary Rhythmic Muscle Contraction-Induced Exercising Blood Flow Variability Measured by Doppler Ultrasound. Open Journal of Molecular and Integrative Physiology, 3, 158-165. https://doi.org/10.4236/ojmip.2013.34021

[19] Osada, T., Ishiyama, M. and Ueno, R. (2019) Voluntary Thigh Muscle Strength with Resection Stump-Dependent Blood Flow and Vasodilation in an Amputated Lower Leg with Total Surface Bearing Prosthesis during Dynamic Knee Extensor: A Case Trial. Open Journal of Therapy and Rehabilitation, 7, 151-169. https://doi.org/10.4236/ojtr.2019.74011

[20] Osada, T. and Rådegran, G. (2016) Difference in Muscle Blood Flow Fluctuations between Dynamic and Static Thigh Muscle Contractions: How to Evaluate Exercise Blood Flow by Doppler Ultrasound. Physical Medicine and Rehabilitation Research, 1, 128. https://doi.org/10.15761/PMRR.1000128

[21] Thomas, K.N., Cotter, J.D., Lucas, S.J., Hill, B.G. and van Rij, A.M. (2015) Reliability of Contrast-Enhanced Ultrasound for the Assessment of Muscle Perfusion in Health and Peripheral Arterial Disease. Ultrasound in Medicine \& Biology, 41, 26-34. https://doi.org/10.1016/j.ultrasmedbio.2014.06.012

[22] Duerschmied, D., Olson, L., Olschewski, M., Rossknecht, A., Freund, G., Bode, C. and Hehrlein, C. (2006) Contrast Ultrasound Perfusion Imaging of Lower Extremities in Peripheral Arterial Disease: A Novel Diagnostic Method. European Heart Journal, 27, 310-315. https://doi.org/10.1093/eurheartj/ehi636

[23] Meneses, A.L., Nam, C.Y., Bailey, T.G., Magee, R., Golledge, J., Hellsten, Y., Keske, M.A., Greaves, K. and Askew C.D. (2018) Leg Blood Flow and Skeletal Muscle Microvascular Perfusion Responses to Submaximal Exercise in Peripheral Arterial Disease. American Journal of Physiology - Heart and Circulatory Physiology, 315, H1425-H1433. https://doi.org/10.1152/ajpheart.00232.2018

[24] Baker, W.B., Li, Z., Schenkel, S.S., Chandra, M., Busch, D.R., Englund, E.K., Schmitz, K.H., Yodh, A.G., Floyd, T.F. and Mohler, E.R. (2017) Effects of Exercise Training on Calf Muscle Oxygen Extraction and Blood Flow in Patients with Peripheral Artery Disease. Journal of Applied Physiology, 123, 1599-1609. https://doi.org/10.1152/japplphysiol.00585.2017 